\title{
Lapatinib-induced inhibition of ovarian function is counteracted by the STAT3 pathway both in vivo and in vitro
}

\author{
QIUYUE LIAO*, XUE FENG*, XI LI, GE CHEN, JING CHEN, BIN YANG, KEZHEN LI and JIHUI AI \\ Department of Gynecology and Obstetrics, Tongji Hospital, Tongji Medical College, \\ Huazhong University of Science and Technology, Wuhan, Hubei 430030, P.R. China
}

Received November 25, 2019; Accepted June 9, 2020

DOI: $10.3892 /$ or.2020.7660

\begin{abstract}
The present study was designed to ascertain whether lapatinib, a tyrosine kinase inhibitor of epidermal growth factor receptor (EGFR), affects ovarian reserve and fertility potential in a mouse model. Female C57BL/6 mice were treated with either vehicle or lapatinib (100 or $200 \mathrm{mg} / \mathrm{kg} / \mathrm{day}$ orally) for 4 weeks, after which body weight, vaginal smears, follicle numbers, serum anti-Müllerian hormone (AMH) levels and mating outcomes were analyzed to assess the ovarian reserve and reproductive function. Slices from the ovaries of 4-week-old mice were cultured with lapatinib $(0,5$ or $10 \mu \mathrm{M})$ for 24 and $48 \mathrm{~h}$, and protein expression levels were assessed to validate the changes in signaling pathways. The results indicated that mice treated with $200 \mathrm{mg} / \mathrm{kg}$ lapatinib showed a slight decrease in body weight compared to those treated with vehicle or $100 \mathrm{mg} / \mathrm{kg}$ lapatinib. There was no statistical difference in estrous cyclicity among the three groups. No significant difference was observed in follicle numbers, $\mathrm{AMH}$ levels, histological morphologies of the ovaries or mating
\end{abstract}

Correspondence to: Professor Jihau Ai or Professor Kezhen Li, Department of Gynecology and Obstetrics, Tongji Hospital, Tongji Medical College, Huazhong University of Science and Technology, 1095 Jiefang Anvenue, Wuhan, Hubei 430030, P.R. China

E-mail: jihuiai@tjh.tjmu.edu.cn

E-mail: tjkeke@126.com

*Joint first authors

Abbreviations: EGF, epidermal growth factor; EGFR, epidermal growth factor receptor; HER2, human epidermal growth factor receptor-2; TKIs, tyrosine kinase inhibitors; COCs, oocyte-cumulus complexes; AMH, anti-Müllerian hormone; PI3K, phosphatidylinositol-3 kinase; AKT, protein kinase B; PTEN, phosphatase and tensin homologue deleted on chromosome ten; MAPK, mitogen-activated protein kinases; ERK, extracellular regulated kinase; JAK, Janus kinase; STAT, signal transducers and activators of transcription; PKA, protein kinase A; CDK, cyclin-dependent kinase

Key words: lapatinib, ovarian reserve, reproductive function, EGF receptor, STAT3 signaling pathway outcomes in the three groups of mice. Western blotting and immunohistochemical staining of the EGF receptor and its main downstream signaling pathways showed decreased phosphorylation of EGFR and mitogen-activated protein kinase (MAPK)3/1 and increased phosphorylation of signal transducers and activators of transcription (STAT) 3 in the lapatinib-treated groups compared to the control group. Our study suggests that lapatinib has little effect on ovarian reserve and reproductive function in a mouse model. This lack of effect of lapatinib on ovarian function may be due to the activation of the STAT3 signaling pathway that counteracts the inhibitory effects of lapatinib on EGF receptors.

\section{Introduction}

Recently, tyrosine kinase inhibitors (TKIs) have been widely developed as targeted anticancer therapeutic drugs. Tyrosine kinases are protein kinases that modulate various cellular signaling pathways including proliferation and differentiation. Overexpression and dysregulation of tyrosine kinases are associated with several types of cancer. TKIs selectively inhibit the phosphorylation of tyrosine kinase receptors and thus suppress the growth, proliferation and differentiation of tumor cells (1).

Although TKIs are thought to be narrowly targeted and relatively low-toxic agents, they are associated with a range of adverse effects. Fatigue, diarrhea, weight loss, hypertension, neurotoxicity, dermatologic and cardiovascular toxicities are common the side effects associated with TKI agents (2). However, there is little research on the toxic effects of TKI agents on ovarian function and reproductive potential and these studies give conflicting results. One case of primary ovarian insufficiency was reported in a 28 -year-old woman treated with imatinib (3), but the cause-and-effect relationship of this case was speculative (4). Two mouse models yielded conflicting results on the impact of imatinib on folliculogenesis $(5,6)$. Thus, more research is needed on the reproductive toxicity of TKIs in women.

Several protein kinases, including protein kinase A (PKA), cyclin-dependent kinase (CDK), mitogen-activated protein kinase (MAPK), human epidermal growth factor receptor (HER) kinases and mammalian target of rapamycin (mTOR) are present in the ovary. Activation or blocking of these protein kinases showed that they are involved in ovarian 
cell proliferation and apoptosis, oocyte maturation, hormone release and response to hormones, and mediating the action of hormones on ovarian functions (7). As TKIs target protein kinase receptors in tumor cells and inhibit their phosphorylation, we speculate that TKIs can also interact with protein kinases in the ovary and influence ovarian function and reproductive potential. However, few data are available on the impact and mechanism of TKIs on ovarian function.

Lapatinib is an oral TKI that selectively binds to the ATP-binding site of the kinase and prevents phosphorylation of the EGF receptor and HER2. Combined with trastuzumab therapy, lapatinib is used in the second-line treatment of advanced or metastatic HER2-positive breast cancer. Breast cancer is one of the most frequently diagnosed cancers among women. In 2015, the incidence of breast cancer among women aged 20 to 39 years was 31.7 per 100,000 women. With advances in systemic therapy, the 5-year survival rate among young women reached $88.5 \%$ between 2010 and 2015 (8). Great attention should be paid to the preservation of ovarian function and fertility in young women who are diagnosed with breast cancer and receive anticancer therapy. As EGF receptors play important roles in oocyte nuclear maturation, cumulus expansion, and ovulation $(9,10)$, it is possible that lapatinib treatment may cause fertility toxicity and have an impact on ovarian function. In vitro culture of porcine oocyte-cumulus complexes (COCs) indicated that lapatinib inhibited the meiotic maturation of COCs (11), but there are no in vivo studies on the effect of lapatinib on ovarian function.

Therefore, we performed in vivo mouse experiments to investigate whether lapatinib could influence ovarian function and fertility potential through the inhibition of EGF receptor tyrosine kinases, and further explored the mechanism of action of lapatinib both in vivo and in vitro.

\section{Materials and methods}

Reagents and antibodies. Lapatinib ditosylate was purchased from Selleck Chemicals. Antibodies were purchased from Cell Signaling Technology, Inc.: Phospho-EGFR (Tyr1068, rabbit monoclonal) (\#3777), PI3K (rabbit monoclonal) (\#4257), phospho-PI3K (Tyr458/Tyr199, rabbit polyclonal) (\#4228), phospho-PTEN (Ser380, rabbit polyclonal) (\#9551), AKT (rabbit monoclonal) (\#4691), phospho-AKT (Ser473, rabbit monoclonal) (\#4060), STAT3 (rabbit monoclonal) (\#4904), phospho-STAT3 (Tyr705, rabbit monoclonal) (\#9145), MEK1/2 (rabbit monoclonal) (\#8727), phospho-MEK1/2 (Ser217/221, rabbit monoclonal) (\#9154), MAPK1/3 (rabbit monoclonal) (\#4695), phospho-MAPK1/3 (Thr202/Tyr204, rabbit monoclonal) (\#4370). EGFR (rabbit polyclonal) (18986-1-AP) and PTEN (rabbit polyclonal) (22034-1-AP) were purchased from Proteintech. GAPDH (rabbit polyclonal) and horseradish peroxidase (HRP)-labeled goat anti-rabbit secondary antibodies were purchased from Servicebio. Antibodies for immunohistochemical staining were purchased from ImmunoWay: Stat3 (phospho Tyr705, rabbit polyclonal) (YP0251) and PI3-kinase p85/p55 (phospho Tyr467/199, rabbit polyclonal) (YP0224); Abcam: Anti-EGFR (phospho Y1068, rabbit monoclonal) (ab40815); and Cell Signaling Technology, Inc.: Phospho-MAPK1/3 (rabbit monoclonal) (\#4370).
Animals and treatments. Five-week-old female and 9-week-old male C57BL/6 mice were purchased from Hubei Provincial Center for Disease Control and Prevention. The animals were provided with ad libitum access to food and water and housed under SFP conditions of controlled temperature $\left(20-25^{\circ} \mathrm{C}\right)$, humidity (40-70\%) and lighting (12 h light-dark cycle). After acclimatization for 1 week, 76 female mice with regular estrous cyclicity were randomized into three groups and administered lapatinib (100 mg/kg, n=22 or $200 \mathrm{mg} / \mathrm{kg}, \mathrm{n}=32$ ) or vehicle (distilled water containing $1 \%$ Tween-80, $\mathrm{n}=22$ ) orally for 4 weeks. The doses for mice was calculated based on the human dose (1,250 mg/day orally) and these doses are also commonly used in mouse models $(12,13)$. In the event that the mice succumbed to the side effects of high dose lapatinib, more mice were randomized into the $200 \mathrm{mg} / \mathrm{kg}$ lapatinib group. Body weights were monitored before and after treatment. Then 44 mice ( $n=12$ in control, $n=12$ in $100 \mathrm{mg} / \mathrm{kg}$ lapatinib, and $\mathrm{n}=20$ in $200 \mathrm{mg} / \mathrm{kg}$ lapatinib) were randomly euthanized and their ovaries were collected for analysis. The remaining control and treated mice were kept for a fertility trial. This animal study was approved by the Ethics Committee of Tongji Hospital, Tongji Medical College, Huazhong University of Science and Technology Institutional (TJ-A20171206).

Estrous cyclicity. Before treatment, vaginal smears were monitored daily for 1 week to exclude mice with irregular estrous cyclicity. At 8:00-9:00 a.m., one drop of PBS was expelled into the vagina, aspirated, and then transferred to a microscope slide. The vaginal samples were analyzed for the predominance of lymphocytes, nucleated epithelial cells or keratinocytes and the estrous cycles of the mice were deemed as regular, irregular, or prolonged estrous, as described previously (14). After treatment for 4 weeks, vaginal smears were again assessed for 1 week to determine the estrous cyclicity.

Ovary histology. Ovaries were fixed in $4 \%$ paraformaldehyde (Servicebio) overnight and embedded in paraffin. After deparaffinization, 5- $\mu \mathrm{m}$-sectioned samples were placed on glass slides and stained with hematoxylin and eosin. Follicles were classified using accepted definitions (15). A primordial follicle was defined as an oocyte surrounded by a single layer of flattened granulosa cells, a primary follicle was surrounded by a single layer of cuboidal granulosa cells, a secondary follicle had at least two layers of cuboidal granulosa cells without an antrum and an antral follicle had an antrum. Follicles were counted every 6th section (every $30 \mu \mathrm{m}$ ) throughout the ovary, and then the follicles in each stage per section were calculated from the total number of follicles per ovary. To avoid double counting of secondary and antral follicles, only follicles with an oocyte nucleus in these stages were counted.

Serum AMH. Blood was collected from the mouse orbital sinus at the time of death and centrifuged at 1,000 x $\mathrm{g}$ for 15 min to extract the serum. The concentration of AMH was quantified with an AMH ELISA kit (Beyotime Institute of Biotechnology) according to the manufacturer's instructions.

Western blotting. Ovarian tissues were lysed in radioimmunoprecipitation assay (RIPA) buffer (Servicebio) 


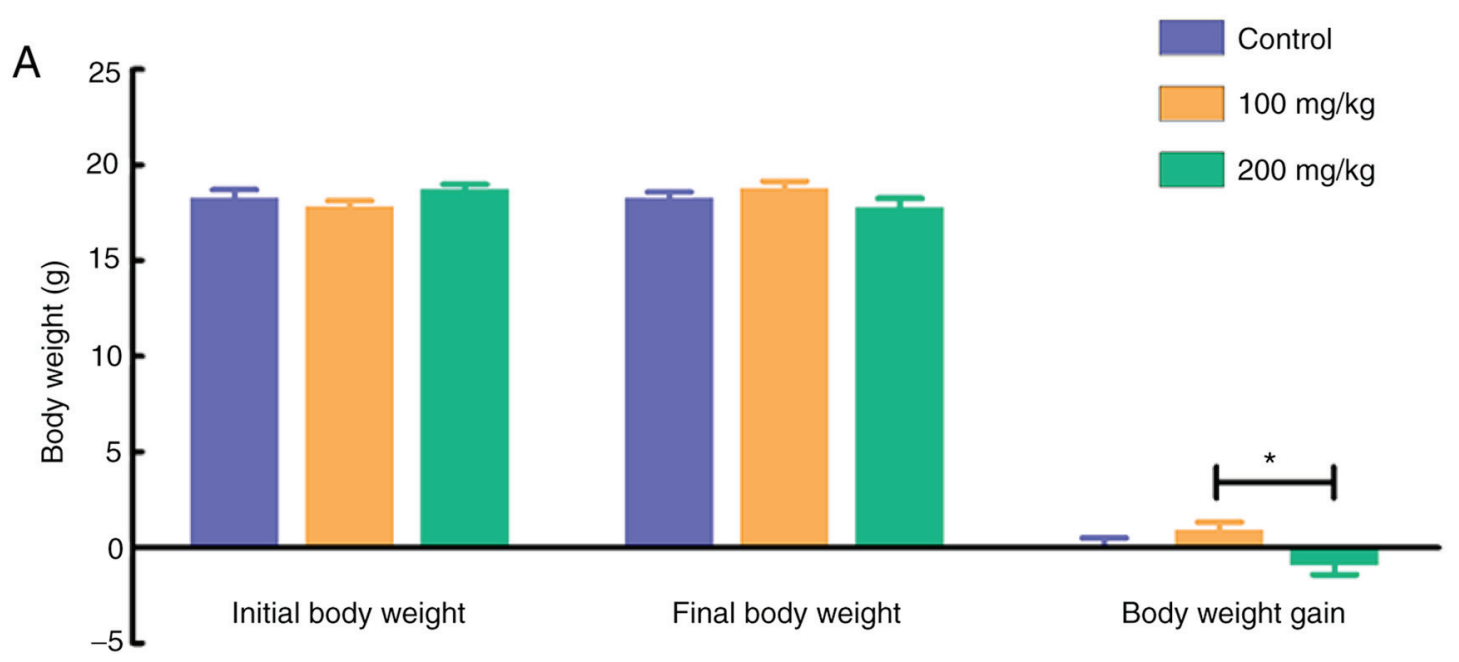

B
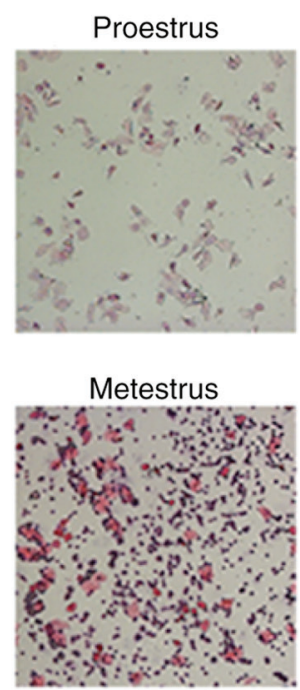
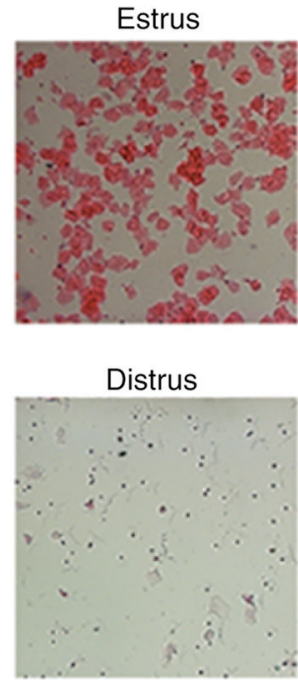

C

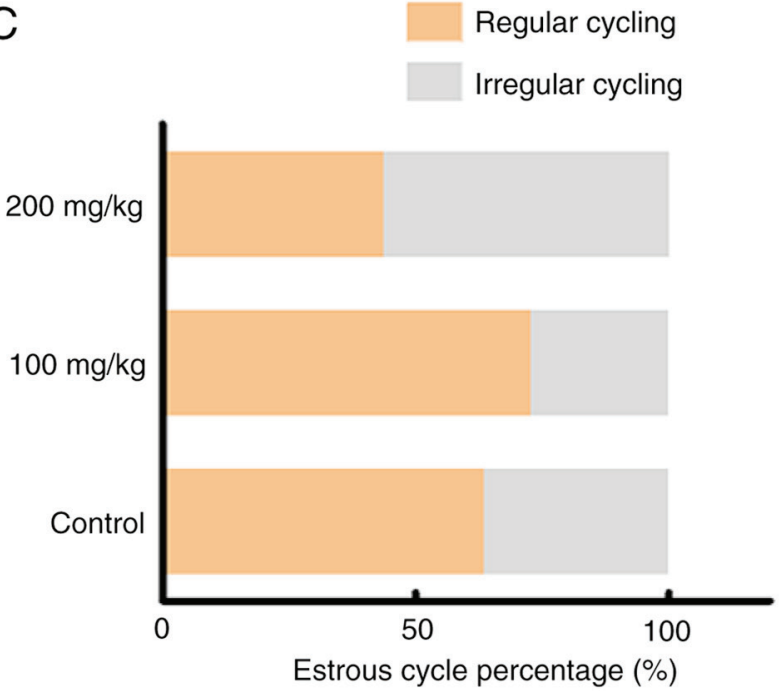

Figure 1. Mouse body weight gain and cyclicity monitored after treatment with lapatinib. (A) Body weight gain of mice was calculated based on the initial and final body weights. ${ }^{*} \mathrm{P}<0.05$, statistical significance. (B) Representative vaginal smears with regular estrous cyclicity under a positive fluorescence microscope (magnification, x100). (C) Percentage of mice with regular and irregular cyclicity after a 4-week treatment with lapatinib. Percentage of mice with regular cyclicity was $63.6 \%$ in the control group, $72.7 \%$ in the $100 \mathrm{mg} / \mathrm{kg}$ group and $43.8 \%$ in the $200 \mathrm{mg} / \mathrm{kg}$ group.

containing $1 \%$ protease inhibitor cocktail (Servicebio) and $1 \%$ phosphorylase inhibitor (Servicebio) for $30 \mathrm{~min}$. Lysates were centrifuged at $12,000 \mathrm{x}$ for $10 \mathrm{~min}$ at $4^{\circ} \mathrm{C}$ and the protein was quantified using a BCA Protein Assay kit (Servicebio). Loading buffer was added to the protein samples. Then $30 \mu \mathrm{g}$ proteins in each lane were separated by $10 \%$ SDS-PAGE before being transferred to polyvinylidene difluoride (PVDF) membranes (Millipore, USA). The membranes were incubated in a blocking buffer (5\% nonfat milk in Tris-buffered saline containing $0.5 \%$ Tween-20) for $1 \mathrm{~h}$ at $37^{\circ} \mathrm{C}$ and then incubated at $4^{\circ} \mathrm{C}$ with primary antibodies overnight. The concentrations of primary antibodies were: EGFR (dilution 1:1,000), phospho-EGFR (Tyr1068, dilution 1:1,000), PI3K (dilution 1:1,000), phospho-PI3K (Tyr458/Tyr199, dilution 1:1,000), PTEN (dilution 1:1,000), phospho-PTEN (Ser380, dilution 1:1,000), AKT (dilution 1:2,000), phospho-AKT (Ser473, dilution 1:2,000), STAT3 (dilution 1:1,000), phospho-STAT3 (Tyr705, dilution 1:1,000), MEK1/2 (dilution 1:1,000), phospho-MEK1/2 (Ser217/221, dilution 1:1,000), MAPK1/3 (dilution 1:1,000), phospho-MAPK1/3 (Thr202/Tyr204, dilution 1:1,000) and GAPDH (dilution 1:1,000). After washing with TBS-Tween $5 \mathrm{~min}$ for three times, the blots were incubated with HRP-labeled goat anti-rabbit secondary antibody (duration $1: 1,000$ ) for $1 \mathrm{~h}$ at $37^{\circ} \mathrm{C}$. Proteins were visualized using a chemiluminescent imager (Syngene) and an ECL kit (Servicebio).

Immunohistochemical staining. Paraffin sections were rehydrated and treated with $0.3 \%$ hydrogen peroxide for $20 \mathrm{~min}$ at room temperature to neutralize endogenous peroxidases. Then sections were immersed in citrate buffer for antigen retrieval in a microwave oven. After incubation in blocking buffer (ready-to-use goat serum, Boster Biological Technology), sections were incubated with primary antibodies at $4^{\circ} \mathrm{C}$ overnight and the secondary antibody (Servicebio at room temperature for $1 \mathrm{~h}$ in a wet box. The concentrations of primary antibodies were: Stat3 (phospho Tyr705, dilution 1:50), PI3-kinase p85/p55 (phospho Tyr467/199, dilution 
A

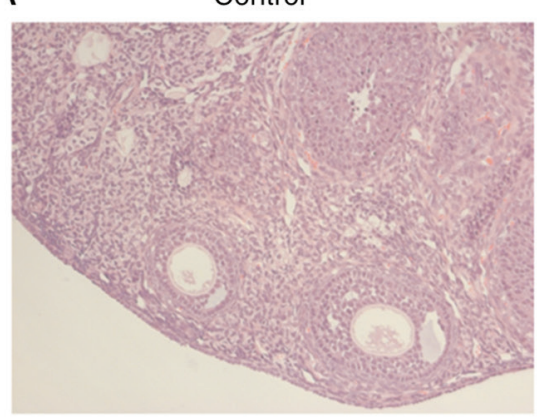

$100 \mathrm{mg} / \mathrm{kg}$

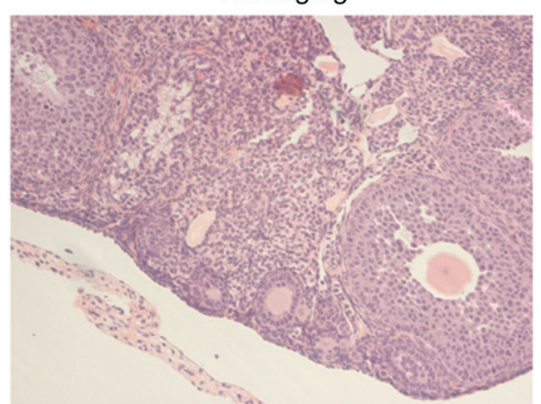

$200 \mathrm{mg} / \mathrm{kg}$

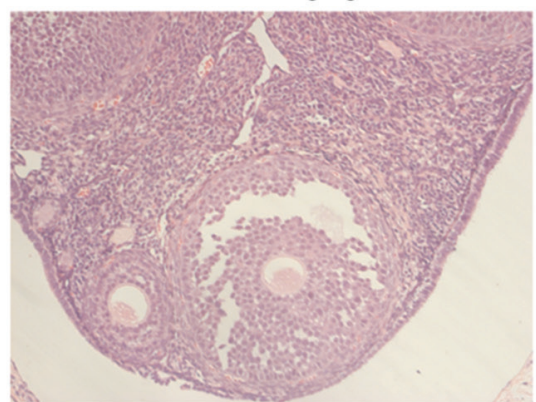

$\mathrm{B}$

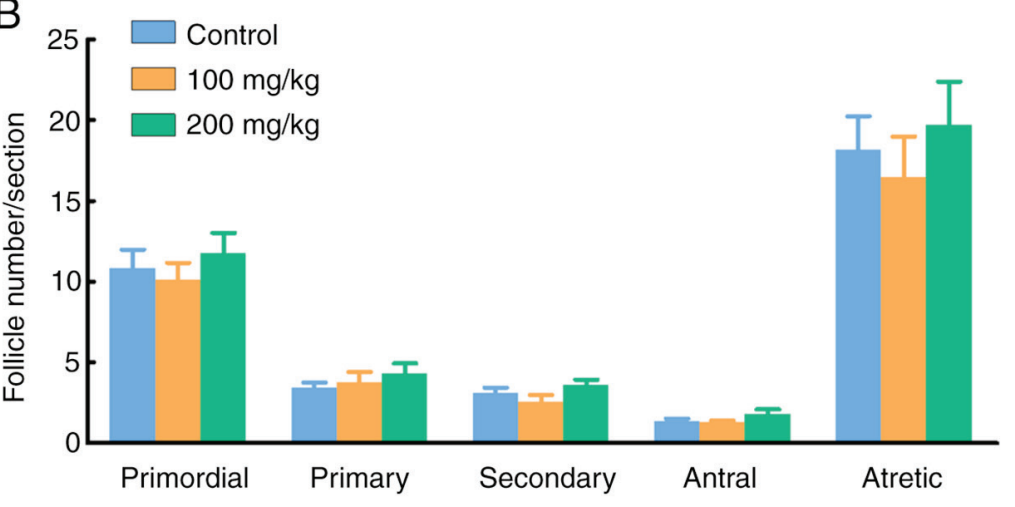

C

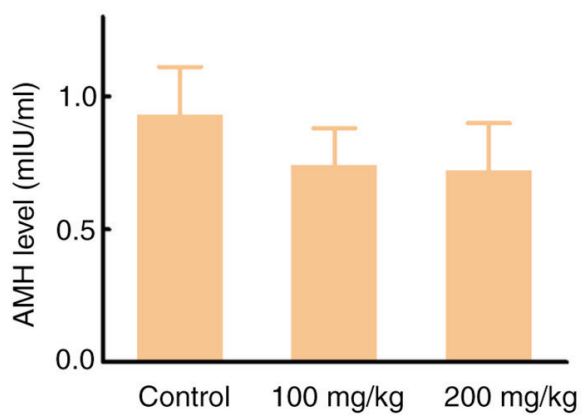

D

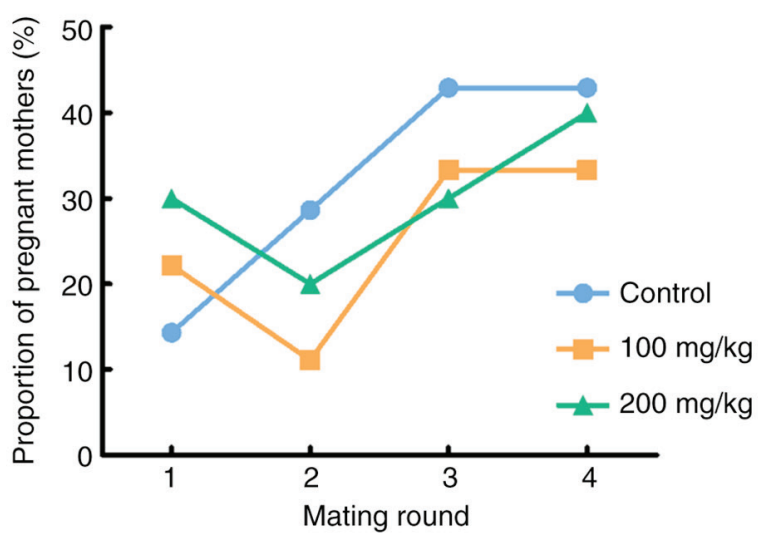

$E$

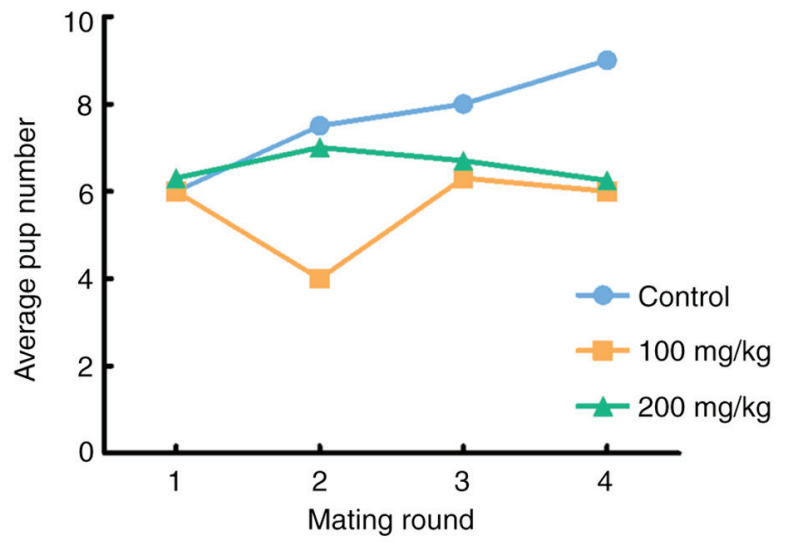

Figure 2. Mouse ovarian reserve and fertility. (A) Representative ovarian histological sections from control mice and lapatinib-treated mice (magnification, x200). (B) Follicle numbers per section of different stages after control, $100 \mathrm{mg} / \mathrm{kg}$ lapatinib and $200 \mathrm{mg} / \mathrm{kg}$ lapatinib treatment. (C) Serum AMH levels in control and lapatinib-treated mice. (D) Pregnancy rate and (E) average pup number after control, $100 \mathrm{mg} / \mathrm{kg}$ lapatinib and $200 \mathrm{mg} / \mathrm{kg}$ lapatinib treatment. AMH, anti-Müllerian hormone.

1:50), phospho-MAPK1/3 (Thr202/Tyr204, dilution 1:50) and anti-EGFR (phospho Y1068, dilution 1:100). All sections were visualized using DAB (Servicebio) and followed by hematoxylin counterstaining. For negative controls, non-immune rabbit serum (Boster Biological Technology) and rabbit mAb IgG (Cell Signaling Technology, Inc.) at the same protein concentration as the primary antibody.

Mating experiments. A total of 32 female mice $(n=10$ in control, $\mathrm{n}=10$ in $100 \mathrm{mg} / \mathrm{kg}$ lapatinib, and $\mathrm{n}=12$ in $200 \mathrm{mg} / \mathrm{kg}$ lapatinib) were used for mating experiments. Two female mice were paired with one proven fertile male mouse 7 days after treatment with lapatinib or vehicle treatment as mentioned above. After 1 week of mating, the females were separated until delivery. Pups were kept with their mothers for 1 week and then separated. One week after breast-feeding, the mice were mated again. The duration of the mating experiment was 6 months, with mating intervals of 5-6 weeks.

Ovarian tissue culture. The ovaries from 4-week-old mice were sliced into 3 or 4 pieces using a micro-scissor (Servicebio) under a stereoscopic microscope (Olympus, Japan). Every 3 or 4 ovary slices were cultured in a $8.0-\mu \mathrm{m}$ Transwell culture insert for 24-well plates (Corning Inc.) under conditions of $5 \% \mathrm{CO}_{2}$ and temperature of $37^{\circ} \mathrm{C}$. Each well was filled with 1,000 $\mu 1$ M199 (Boster Biological Technology) supplemented with 5\% fetal bovine serum (FBS) (Tianhang Biotechnology), 1\% insulin-transferrin-sodium 

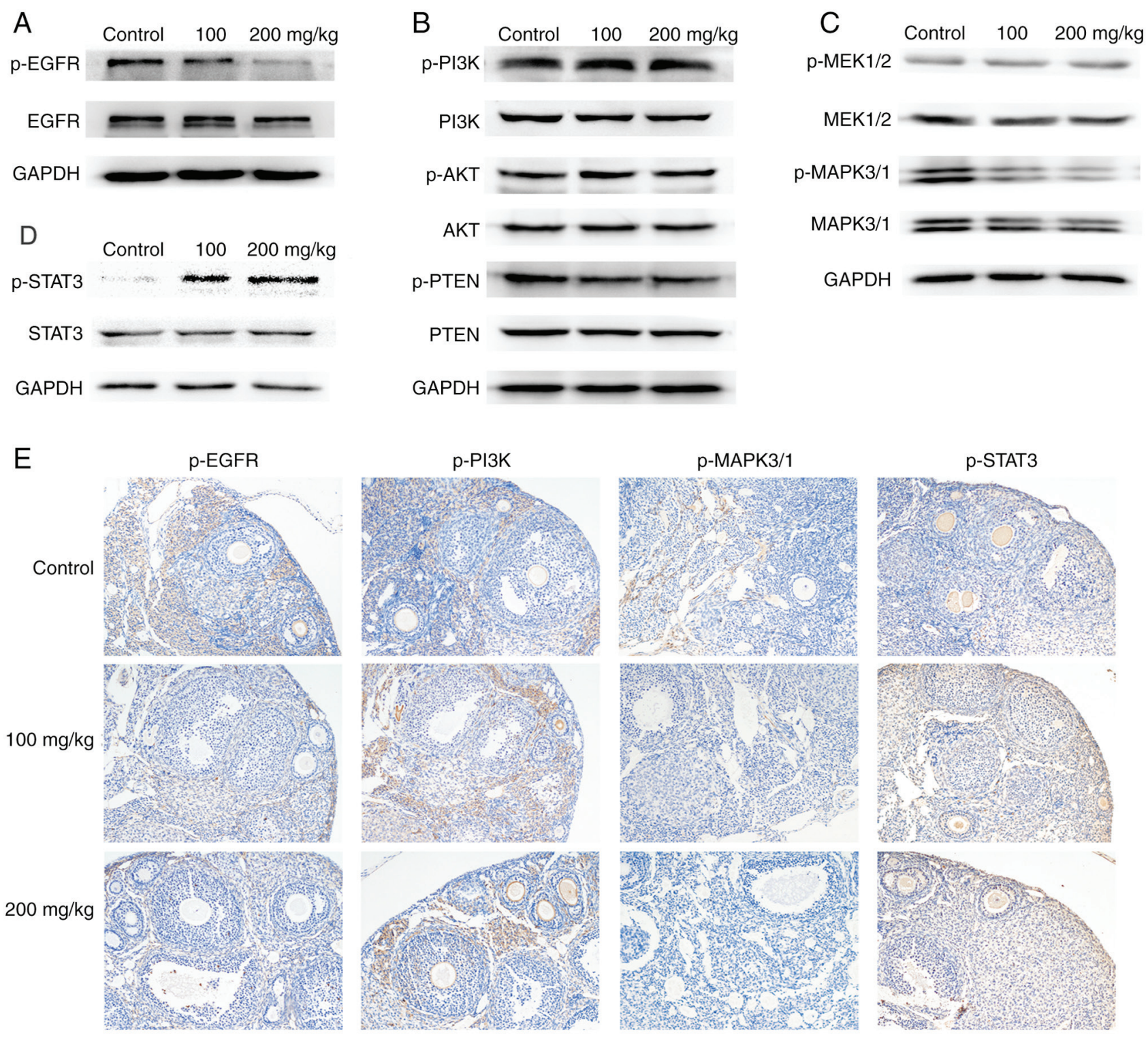

Figure 3. Western blot analysis and immunohistochemical staining of the EGFR signaling pathway in vivo. Western blotting of ovaries treated with control and lapatinib in vivo showed (A) decreased phosphorylation of EGFR (p-EGFR) and (C) MAPK3/1 in the lapatinib-treated groups, and (D) increased phosphorylation of STAT3 in the lapatinib-treated groups. (B) No obvious difference was noted in the phosphorylation of the PI3K/AKT pathway. (E) Immunohistochemical staining confirmed these results (magnification, x100). EGFR, epidermal growth factor receptor; MAPK, mitogen-activated protein kinase; PI3K, phosphatidylinositol-3 kinase; AKT, protein kinase B; PTEN, phosphatase and tensin homologue deleted on chromosome ten; STAT, signal transducers and activators of transcription.

(ITS) (Sigma-Aldrich; Merck KGaA), $100 \mathrm{U} / \mathrm{ml}$ penicillin G and $100 \mu \mathrm{g} / \mathrm{ml}$ streptomycin (Servicebio), and $100 \mu \mathrm{IU} / \mathrm{ml}$ follicle-stimulating hormone (FSH) (Livzon). After $24 \mathrm{~h}$ of culture, either vehicle (1\% DMSO) or $5 \mu \mathrm{M}$ or $10 \mu \mathrm{M}$ lapatinib was added to the medium and the samples were cultured for a further 24 and $48 \mathrm{~h}$. This dose range covers the human dose of $1,500 \mathrm{mg} /$ day $(\sim 5.6 \mathrm{mM})(11)$. The tissue culture at each culture time and drug concentration was replicated for at least 3 times.

Statistical analysis. All data are presented as the means \pm standard deviation (SD) or standard error of the mean (SEM). Statistical analyses were performed using GraphPad Prism 5.0 (GraphPad Software, USA) and Statistical Package for Social Sciences 18.0 (SPSS, Inc.). Body weights, follicle numbers, AMH levels and mating outcomes were analyzed by one-way
ANOVA, and pairwise comparisons were performed using Student-Newman-Keuls (SNK) post hoc test. Estrous cyclicity was analyzed by Chi-squared test. $\mathrm{P}<0.05$ was considered statistically significant.

\section{Results}

Effect of lapatinib on the general condition of the mice. To evaluate the effects of lapatinib on the general health of the mice, 76 6-week-old female mice were randomized into three groups and treated with either lapatinib $(100 \mathrm{mg} / \mathrm{kg}$, $\mathrm{n}=22$ or $200 \mathrm{mg} / \mathrm{kg}, \mathrm{n}=32)$ or vehicle $(\mathrm{n}=22)$ for 4 weeks. The mice treated with $200 \mathrm{mg} / \mathrm{kg}$ lapatinib showed a significant decrease in body weight (Fig. 1A, Table SI) compared to the $100 \mathrm{mg} / \mathrm{kg}$ group. The percentage of irregular estrous cycling was higher in the mice treated with $200 \mathrm{mg} / \mathrm{kg}$ lapatinib than 
A
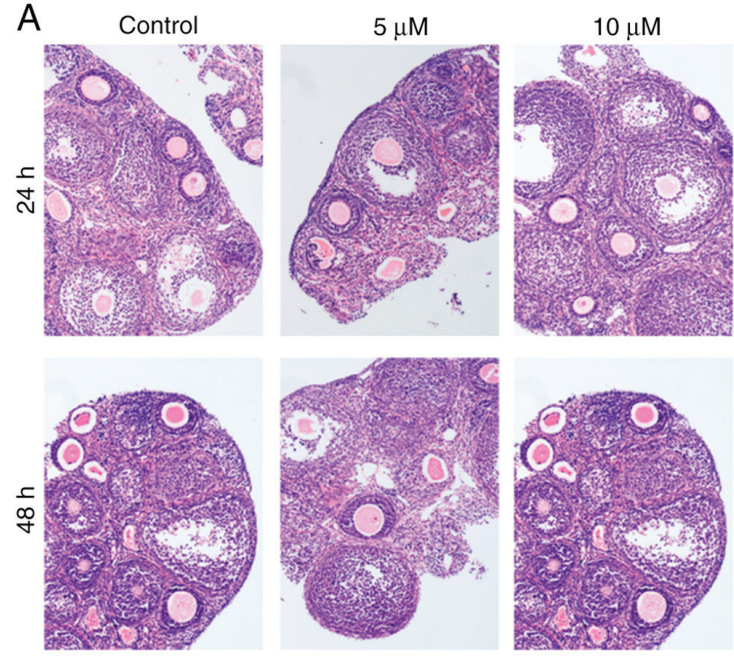

C

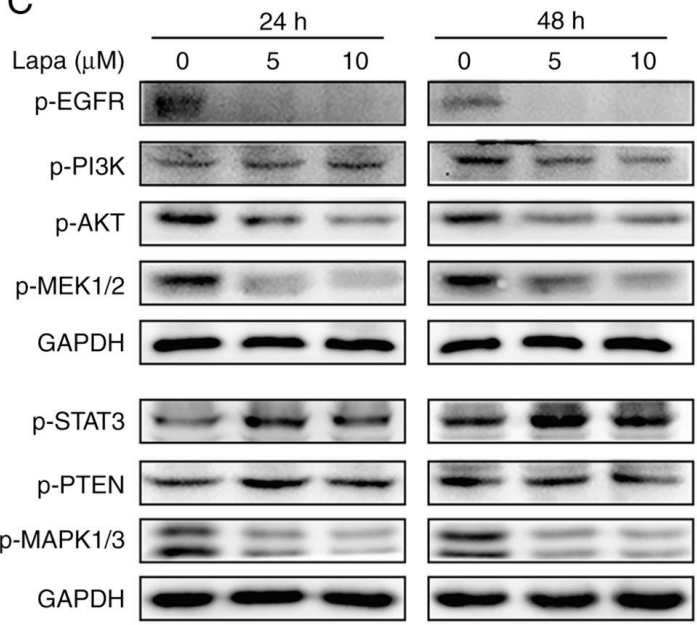

B
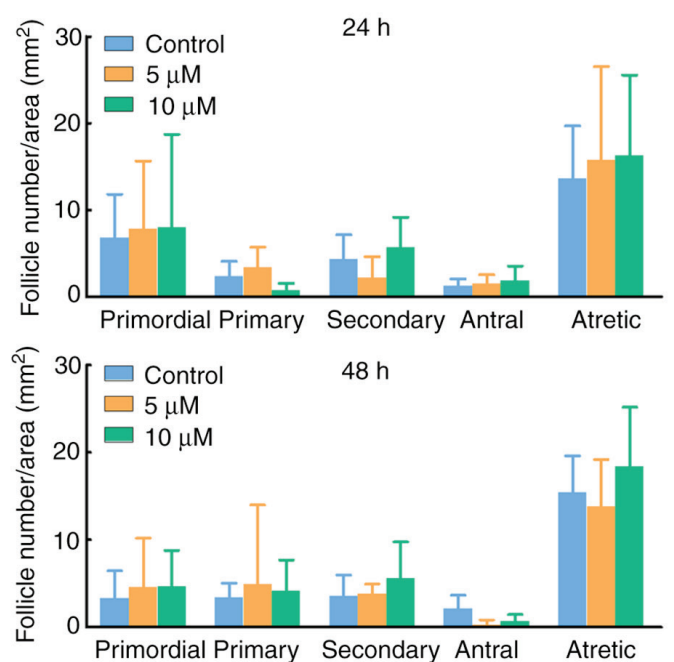

D

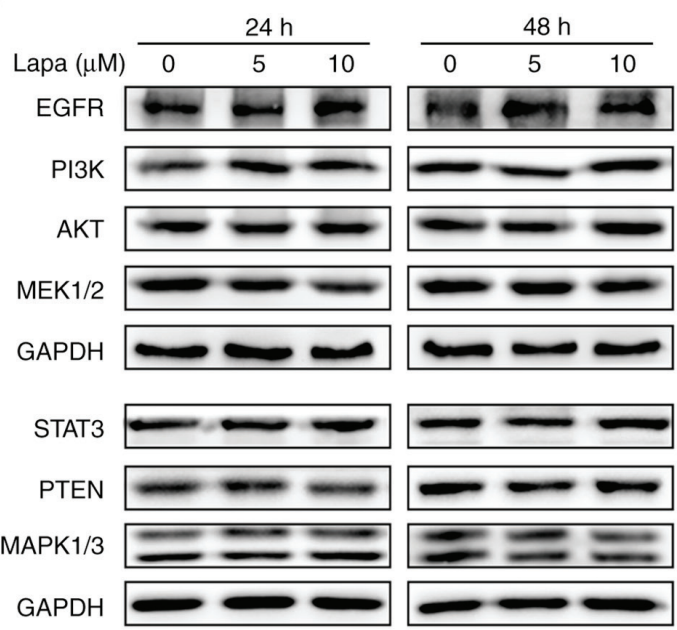

Figure 4. Histological assessment and western blot analysis of the ovarian slices in vitro. (A) Hematoxylin and eosin staining of ovarian slices cultured with or without lapatinib $(5$ and $10 \mu \mathrm{M})$ for 24 and $48 \mathrm{~h}$ (magnification, x100). (B) Follicle numbers per area $\left(\mathrm{mm}^{2}\right)$ of ovarian slices treated for 24 and $48 \mathrm{~h}$. (C and D) Lapatinib (Lapa) inhibits the phosphorylation of EGFR (p-EGFR) and the PI3K/AKT and MAPK/ERK pathways; this inhibition was counteracted by activation of STAT3. EGFR, epidermal growth factor receptor; PI3K, phosphatidylinositol-3 kinase; AKT, protein kinase B; MAPK, mitogen-activated protein kinases; ERK, extracellular regulated kinase; STAT, signal transducers and activators of transcription; PTEN, phosphatase and tensin homologue deleted on chromosome ten.

in the control or $100 \mathrm{mg} / \mathrm{kg}$ treatment groups (Fig. 1B and C), but the difference was not statistically significant.

Effect of lapatinib on ovarian reserve and fertility. To investigate the effect of lapatinib on ovarian reserve, a total of 44 mice in three groups ( $\mathrm{n}=12$ in control, $\mathrm{n}=12 \mathrm{in} 100 \mathrm{mg} / \mathrm{kg}$ lapatinib, and $\mathrm{n}=20 \mathrm{in} 200 \mathrm{mg} / \mathrm{kg}$ lapatinib) were sacrificed after treatment and ovarian morphology and anti-Müllerian hormone $(\mathrm{AMH})$ were measured. Ten ovarian samples in each group were randomly selected for ovary histology assessment. No apparent difference was observed in the histological morphology of the ovaries (Fig. 2A). Follicle numbers of the different stages were similar among the three groups (Fig. 2B, Table SII), which indicated that lapatinib did not influence oocyte maturation, follicle activation, or follicle apoptosis. Serum AMH levels showed a slight decrease in the lapatinib-treated groups compared with the control group, however, the data were not statistically significant (Fig. 2C, Table SIII). The remaining 32 female mice were kept for mating experiment. During this procedure, 6 mice died and the reason was unknown (may be the side effects of drugs or gavage). Ultimately 26 living mice were included in the analysis ( $\mathrm{n}=7$ in control, $\mathrm{n}=9 \mathrm{in} 100 \mathrm{mg} / \mathrm{kg}$ lapatinib, and $\mathrm{n}=10$ in $200 \mathrm{mg} / \mathrm{kg}$ lapatinib). The results showed that the pregnancy rate was not significantly influenced by the lapatinib treatment (Fig. 2D, Table SIV). Although the average number of pups showed a decrease in the lapatinib-treated groups, the data were not statistically significant (Fig. 2E, Table SV).

Effect of lapatinib on the EGF receptor signaling pathway. Theoretically, the EGFR and HER2 inhibitor lapatinib can influence ovarian function and reproductive potential. Therefore, to explore the mechanism for the lack of reproduction toxicity of lapatinib in our experiments, ovaries of the sacrificed mice after the 4-week treatment were collected to evaluate the expression levels of the EGF receptor and its downstream signaling pathways using western blotting and immunohistochemical staining. We found that the phosphorylation site of the EGFR receptor, the target of lapatinib, showed significantly decreased phosphorylation in the lapatinib-treated groups compared to the control group (Fig. 3A and E). We also found decreased 
phosphorylation of MAPK3/1 and increased phosphorylation of STAT3 in the lapatinib-treated groups compared to the control group (Fig. 3C, D and E). However, no obvious variation was noted in the phosphorylation of the PI3K/AKT pathway (Fig. 3B). This result indicated that lapatinib was able to inhibit the activation of the EGF receptor and further inhibit its downstream MAPK/ERK pathway in mouse ovary. However, activation of the STAT3 pathway may counteract these inhibitory effects. Therefore, no apparent effect was observed in either the estrous cyclicity or the ovarian reserve of the mice.

In vitro effect of lapatinib on ovarian tissue and oocytes. As the targets of lapatinib are found throughout the body, to exclude the global effects of lapatinib on the whole body and to confirm the direct effects of lapatinib on the ovaries and oocytes, in vitro experiments were performed using ovarian tissue slices. Ovarian tissues were cultured with or without lapatinib (5 and $10 \mu \mathrm{M}$ ) for 24 and $48 \mathrm{~h}$. There were no obvious differences in the morphological assessment (Fig. 4A) and follicle counting (Fig. 4B) of the ovarian slices after treatment. Western blotting experiments to examine the EGF receptor and the downstream pathways showed similar results as those noted with the in vivo experiments (Fig. $4 \mathrm{C}$ and D). The phosphorylation of EGFR, MEK1/2, MAPK3/1, PI3K and AKT decreased in the lapatinib-treated groups, and phosphorylation of STAT3 increased in the lapatinib-treated groups compared to the control group. These results indicated that the inhibition of the EGFR and the PI3K and MAPK pathways by lapatinib may be counteracted by the activation of the STAT3 signaling pathway.

\section{Discussion}

Lapatinib is an oral dual tyrosine kinase inhibitor that selectively targets the tyrosine kinase domain of the epidermal growth factor (EGF) and human epidermal growth factor receptor-2 (HER2) receptors. Commonly reported side effects of lapatinib include diarrhea, skin rash, and headache (16), but the toxic effects on gonadal function and fertility are uncertain. Our study is the first to investigate the effects of lapatinib on ovarian function and reproductive potential in a mouse model. We performed in vivo experiments to study the estrous cyclicity, ovarian reserve and fertility potential of female mice after a 4-week lapatinib treatment, and the results showed that lapatinib was well tolerated and had little effect on ovarian function and fertility in mice. Western blotting indicated that while lapatinib inhibited ovarian EGF receptors, the activation of the signal transducers and activators of transcription (STAT) 3 signaling pathway may counteract this inhibitory effect.

In the present study, mice were treated with either lapatinib or vehicle. The body weight of the mice treated with $200 \mathrm{mg} / \mathrm{kg}$ lapatinib was significantly lower than that of the mice treated with $100 \mathrm{mg} / \mathrm{kg}$ or the control mice. Weight loss is a side effect commonly noted in tyrosine kinase inhibitors (TKIs) (2). Although we observed slight decreases in estrous cycling, serum AMH levels and the average number of pups in the lapatinib-treated groups, there were no statistical differences among the three groups. These results demonstrated that lapatinib had little effect on the endocrine system, oocyte maturation, ovarian reserve or fertility of female mice.

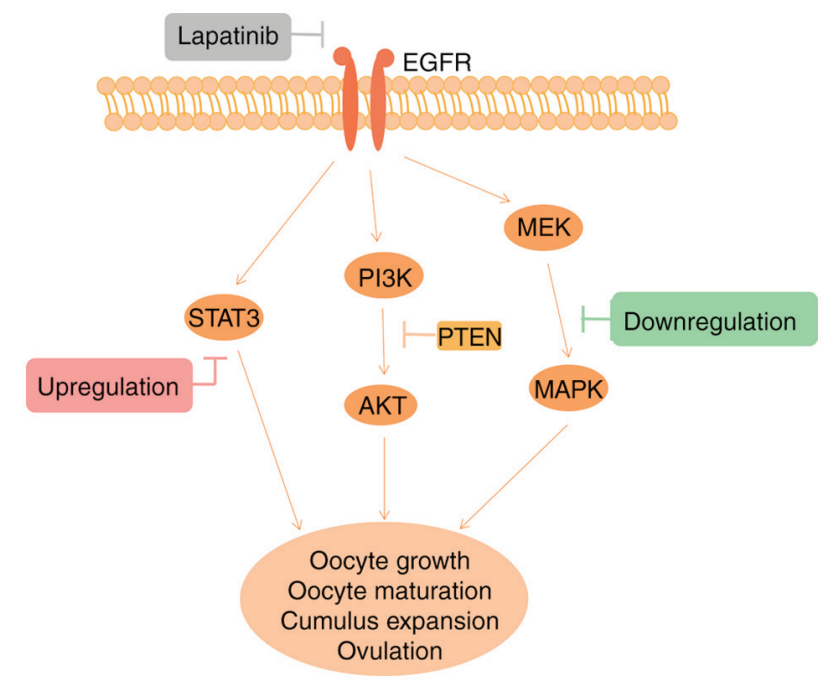

Figure 5. Schematic of STAT3 counteraction of EGFR inhibition by lapatinib. Lapatinib successfully inhibits EGFR activity and the downstream pathways in mouse ovaries, yet the inhibitory effects may be counteracted by the activation of STAT3. Thus, little effect of lapatinib treatment was conferred on oocyte maturation, ovarian reserve and reproductive function in mice. EGFR, epidermal growth factor receptor; STAT, signal transducers and activators of transcription; PI3K, phosphatidylinositol-3 kinase; AKT protein kinase B; PTEN, phosphatase and tensin homologue deleted on chromosome ten; MEK, mitogen-activated extracellular signal-regulated kinase.

To the best of our knowledge, there are no studies on the influence of lapatinib on human ovarian function. Nevertheless, some in vitro animal studies have shown a relationship between EGFR inhibitors and oocyte maturation. In vitro culture of bovine oocyte-cumulus complexes (COCs) showed that the EGFR inhibitor AG1478 promoted oocyte arrest at the GV stage (17). In rat follicles, AG1478 blocked the LH stimulation of EGFR and inhibited oocyte maturation and cumulus expansion (18). The inhibitory effect of lapatinib on porcine COCs was similar to the effect of AG1478, which inhibited oocyte maturation and reduced cumulus expansion (11). It is therefore interesting that lapatinib showed no inhibitory effects on the ovary in our in vivo study.

To investigate the mechanisms behind the lack of in vivo inhibition of ovarian function in our study, we conducted western blotting and immunohistochemical staining of EGFR and its downstream signaling pathways in samples from our control and lapatinib-treated ovaries. Activation of EGFR promotes several signaling pathways, including phosphatidylinositol-3 kinase (PI3K)/protein kinase B (AKT), mitogen-activated protein kinases (MAPK)/extracellular regulated kinase (ERK) and Janus kinase (JAK)/signal transducers and activators of transcription (STAT) pathways (9). Our study showed that lapatinib targeted ovarian EGF receptors and inhibited the phosphorylation of EGFR proteins, which further downregulated the MAPK/ERK signaling pathway. However, the STAT3 pathway was upregulated following lapatinib treatment. To exclude the potential effects of lapatinib on other tissues and organs in the body and verify the outcome, we cultured ovary slices in vitro with or without lapatinib. The western blotting results of the ovary slices also showed inhibition of EGFR and the PI3K and MAPK pathways, and activation of the STAT3 pathway following lapatinib treatment. 
Previous evidence has shown that both the MAPK/ERK and JAK/STAT pathways are necessary for oocyte maturation and function in the mouse. Disruption of MAPK3/1 in mouse granulosa cells resulted in complete infertility in vivo: No oocytes matured, no cumulus-oocyte complex (COC) expanded, and no ovulation occurred (19). Inhibition of MAPK3/1 further demonstrated the role of the MAPK pathway in oocyte meiosis, cumulus expansion and ovulation in mice $(20,21)$. Similarly, STAT3 has been proven to be essential for the formation and function of follicles (22). Activation of p-STAT3 was found to be involved in meiotic spindle assembly and chromosome segregation in mouse oocytes (23), and STAT3 activation was able to stimulate the progression of meiosis (24). In vitro maturation of COCs supplemented with STAT3 inhibitor stattic was found to block cumulus expansion (25). Based on these findings, we speculated that the inhibition of EGFR and MAPK1/3 in the ovary had been negated by the upregulation of STAT3, thus no effects of lapatinib treatment were noted on ovarian reserve or fertility potential in vivo (Fig. 5).

In fact, STAT3 signaling upregulation has been shown in clinical and laboratory tests to be one of the mechanisms of resistance in some anti-EGFR therapeutics in several cancer types (26). Clinical evidence showed that patients who are resistant to EGFR therapeutics such as gefitinib, cetuximab and lapatinib showed increased activity of STAT3 in their tumor tissues (26-28). Cell line studies of hepatoma, colon and head and neck cancers also showed that the level of STAT3 phosphorylation was correlated with the efficacy of anti-EGFR agents $(27,29,30)$. Furthermore, many studies have shown that combining anti-EGFR agents with agents that block STAT3 activity could overcome this drug resistance (31-34). These results strongly suggest that STAT3 signaling counteracts the therapeutic effects of EGFR inhibition in tumors. However, to the best of our knowledge, there are no data on STAT3 counteractive effects in nontumor tissues. Our study is the first to show that the inhibitory effects of EGFR may be counteracted by the STAT3 signaling pathway in healthy mouse ovaries. The mechanism of the STAT3 regulation of EGFR may be similar to that noted in tumor resistance to TKIs, or it may be due to some other kinase-substrate relationships in mouse ovary. The exact mechanism needs to be further explored.

Still, our study has some limitations. The major one was that there was no experimental data on the molecular mechanism between the phosphorylation of STAT3 and ovarian reserve. As this molecular mechanism has been demonstrated in the literature, we gave less effort on this and we mainly focused on the impact of lapatinib on ovary and fertility. Further analysis is needed to make our conclusion more reliable.

In conclusion, our study demonstrated that although lapatinib can target EGF receptors and inhibit downstream pathways in ovaries, the inhibitory effects of lapatinib may be counteracted by activation of the STAT3 signaling pathway. Thus, there was little effect of lapatinib on oocyte maturation, follicle count, ovarian reserve or reproductive function in lapatinib-treated mice. Our data are valuable to young women who receive lapatinib therapy and wish to preserve their fertility. However, large scale clinical data are still needed to confirm that lapatinib does not impair fertility.

\section{Acknowledgements}

We thank the Laboratory Animal Center of Tongji Hospital for the animal handling. We thank the Department of Gynecology and Obstetrics of Tongji Hospital for providing a good experimental platform.

\section{Funding}

This study was supported by the National Natural Science Foundation of China (81802896), Natural Science Foundation of Hubei Province (2017CFB800), Hubei Province Health and Family Planning Scientific Research Project (WJ2017Z013, WJ2019M127) and National Key Research and Development Program (2018YFC1002103, 2019YFC1005200 and 2019YFC1005202).

\section{Availability of data and materials}

The datasets supporting the current study are available on request: Please contact tjkeke@126.com or jihuiai@tjh.tjmu. edu.cn.

\section{Authors' contributions}

QL and XF performed the experiments, carried out data analysis, and drafted the article. XL, GC, JC and BY coordinated the research and experiments. KL and JA were responsible for the study design and manuscript revision. All authors read and approved the final manuscript.

\section{Ethics approval and consent to participate}

Our procedures were performed in accordance with the Guide for the Care and Use of Laboratory Animals. The experiment was proved by the Ethics Committee of Tongji Hospital, Tongji Medical College, Huazhong University of Science and Technology Institutional (TJ-A20171206).

\section{Patient consent for publication}

Not applicable.

\section{Competing interests}

The authors declare that they have no competing interests.

\section{References}

1. Johnson LN: Protein kinase inhibitors: Contributions from structure to clinical compounds. Q Rev Biophys 42: 1-40, 2009.

2. Dy GK and Adjei AA: Understanding, recognizing, and managing toxicities of targeted anticancer therapies. CA Cancer J Clin 63: 249-279, 2013.

3. Christopoulos C, Dimakopoulou V and Rotas E: Primary ovarian insufficiency associated with imatinib therapy. N Engl J Med 358: 1079-1080, 2008.

4. Malozowski S, Nelson L and Calis KA: More on ovarian insufficiency with imatinib. N Engl J Med 358: 2648, 2008.

5. Schultheis B, Nijmeijer BA, Yin H, Gosden RG and Melo JV: Imatinib mesylate at therapeutic doses has no impact on folliculogenesis or spermatogenesis in a leukaemic mouse model. Leuk Res 36: 271-274, 2012. 
6. Asadi-Azarbaijani B, Santos RR, Jahnukainen K, Braber S, van Duursen MBM, Toppari J, Saugstad OD, Nurmio M and Oskam IC: Developmental effects of imatinib mesylate on follicle assembly and early activation of primordial follicle pool in postnatal rat ovary. Reprod Biol 17: 25-33, 2017.

7. Sirotkin AV, Makarevich AV and Grosmann R: Protein kinases and ovarian functions. J Cell Physiol 226: 37-45, 2011.

8. Guo F, Kuo YF, Shih YCT, Giordano SH and Berenson AB: Trends in breast cancer mortality by stage at diagnosis among young women in the United States. Cancer 124: 3500-3509, 2018.

9. Richani D and Gilchrist RB: The epidermal growth factor network: Role in oocyte growth, maturation and developmenta competence. Hum Reprod Update 24: 1-14, 2018.

10. Conti M, Hsieh M, Park JY and Su YQ: Role of the epidermal growth factor network in ovarian follicles. Mol Endocrinol 20: 715-723, 2006.

11. Nagyova E, Nemcova L, Mlynarcikova A, Scsukova S and Kalous J: Lapatinib inhibits meiotic maturation of porcine oocyte-cumulus complexes cultured in vitro in gonadotropin-supplemented medium. Fertil Steril 99: 1739-1748, 2013.

12. Ma Z, Parris AB, Xiao Z, Howard EW, Kosanke SD, Feng X and Yang X: Short-term early exposure to lapatinib confers lifelong protection from mammary tumor development in MMTV-erbB-2 transgenic mice. J Exp Clin Cancer Res 36: 6, 2017.

13. Spector NL, Robertson FC, Bacus S, Blackwell K, Smith DA, Glenn K, Cartee L, Harris J, Kimbrough CL, Gittelman M, et al: Lapatinib plasma and tumor concentrations and effects on HER receptor phosphorylation in tumor. PLoS One 10: e0142845, 2015.

14. Nelson JF, Felicio LS, Randall PK, Sims C and Finch CE: A longitudinal study of estrous cyclicity in aging C57BL/6J mice: I. Cycle frequency, length and vaginal cytology. Biol Reprod 27: 327-339, 1982.

15. Pedersen $\mathrm{T}$ and Peters $\mathrm{H}$ : Proposal for a classification of oocytes and follicles in the mouse ovary. J Reprod Fertil 17: 555-557, 1968.

16. Voigtlaender M, Schneider-Merck T and Trepel M: Lapatinib. Recent Results Cancer Res 211: 19-44, 2018.

17. da Rosa PRA, De Cesaro MP, Pereira Dau AM, Duggavathi R, Bordignon V and Gonçalves PBD: Reversible meiotic arrest of bovine oocytes by EGFR inhibition and follicular hemisections. Theriogenology 99: 53-62, 2017.

18. Ashkenazi H, Cao X, Motola S, Popliker M, Conti M and Tsafriri A: Epidermal growth factor family members: Endogenous mediators of the ovulatory response. Endocrinology 146: 77-84, 2005.

19. Fan HY, Liu Z, Shimada M, Sterneck E, Johnson PF, Hedrick SM and Richards JS: MAPK3/1 (ERK1/2) in ovarian granulosa cells are essential for female fertility. Science 324: 938-941, 2009.

20. Siddappa D, Beaulieu É, Gévry N, Roux PP, Bordignon V and Duggavathi R: Effect of the transient pharmacological inhibition of Mapk3/1 pathway on ovulation in mice. PLoS One 10 e0119387, 2015.

21. Su YQ, Denegre JM, Wigglesworth K, Pendola FL, O'Brien MJ and Eppig JJ: Oocyte-dependent activation of mitogen-activated protein kinase $(E R K 1 / 2)$ in cumulus cells is required for the maturation of the mouse oocyte-cumulus cell complex. Dev Biol 263: 126-138, 2003.
22. Sobinoff AP, Sutherland JM and Mclaughlin EA: Intracellular signalling during female gametogenesis. Mol Hum Reprod 19: 265-278, 2013.

23. Haraguchi S, Ikeda M, Akagi S and Hirao Y: Dynamic changes in pStat 3 are involved in meiotic spindle assembly in mouse oocytes. Int J Mol Sci 21: 1220, 2020.

24. Lee HS, Kim KH, Kim EY, Lee SY, Ko JJ and Lee KA: Obox4-silencing-activated STAT3 and MPF/MAPK signaling accelerate nuclear membrane breakdown in mouse oocytes. Reproduction 151: 369-378, 2016.

25. Tscherner A, Brown AC, Stalker L, Kao J, Dufort I, Sirard MA and LaMarre J: STAT3 signaling stimulates miR-21 expression in bovine cumulus cells during in vitro oocyte maturation. Sci Rep 8: 11527, 2018.

26. Zulkifli AA, Tan FH, Putoczki TL, Stylli SS and Luwor RB: STAT3 signaling mediates tumour resistance to EGFR targeted therapeutics. Mol Cell Endocrinol 451: 15-23, 2017.

27. Dobi E, Monnien F, Kim S, Ivanaj A, N'Guyen T, Demarchi M, Adotevi O, Thierry-Vuillemin A, Jary M, Kantelip B, et al: Impact of STAT3 phosphorylation on the clinical effectiveness of anti-EGFR-based therapy in patients with metastatic colorectal cancer. Clin Colorectal Cancer 12: 28-36, 2013.

28. Haura EB, Sommers E, Song L, Chiappori A and Becker A: A pilot study of preoperative gefitinib for early-stage lung cancer to assess intratumor drug concentration and pathways mediating primary resistance. J Thorac Oncol 5: 1806-1814, 2010.

29. Li Q, Zhang D, Chen X, He L, Li T, Xu X and Li M: Nuclear PKM2 contributes to gefitinib resistance via upregulation of STAT3 activation in colorectal cancer. Sci Rep 5: 16082, 2015

30. Bonner JA, Yang ES, Trummell HQ, Nowsheen S, Willey CD and Raisch KP: Inhibition of STAT-3 results in greater cetuximab sensitivity in head and neck squamous cell carcinoma. Radiother Oncol 99: 339-343, 2011.

31. Dowlati A, Nethery D and Kern JA: Combined inhibition of epidermal growth factor receptor and JAK/STAT pathways results in greater growth inhibition in vitro than single agent therapy. Mol Cancer Ther 3: 459-463, 2004.

32. Sen M, Joyce S, Panahandeh M, Li C, Thomas SM, Maxwell J, Wang L, Gooding WE, Johnson DE and Grandis JR: Targeting stat 3 abrogates EGFR inhibitor resistance in cancer. Clin Cancer Res 18: 4986-4996, 2012.

33. Wen W, Wu J, Liu L, Tian Y, Buettner R, Hsieh MY, Horne D, Dellinger TH, Han ES, Jove R and Yim JH: Synergistic anti-tumor effect of combined inhibition of EGFR and JAK/STAT3 pathways in human ovarian cancer. Mol Cancer 14: 100, 2015.

34. Lee HJ, Zhuang G, Cao Y, Du P, Kim HJ and Settleman J: Drug resistance via feedback activation of Stat 3 in oncogene-addicted cancer cells. Cancer Cell 26: 207-221, 2014.

This work is licensed under a Creative Commons Attribution-NonCommercial-NoDerivatives 4.0 International (CC BY-NC-ND 4.0) License. 